\title{
Serum undercarboxylated osteocalcin levels are related to bone disease in hemodialysis and peritoneal dialysis patients
}

\author{
Medine Alpdemir $^{1} \odot$, Vildan Fidancı ${ }^{2} \oplus$, Mehmet Fatih Alpdemir $^{1} \oplus$, Alper Azak $^{3} \odot$, Gülsevim \\ Saydam $^{4} \oplus$, Murat Duranay $^{5} \odot$, Doğan Yücel ${ }^{2} \odot$
}

${ }^{1}$ Department of Clinical Biochemistry, Balıkesir State Hospital, Balıkesir, Turkey

${ }^{2}$ Department of Clinical Biochemistry, Ankara Training and Research Hospital, Ankara, Turkey

${ }^{3}$ Department of Nephrology, Balıkesir Atatürk City Hospital, Balıkesir, Turkey

${ }^{4}$ Department of Clinical Biochemistry, Türkiye Yüksek İhtisas Training and Research Hospital, Ankara, Turkey

${ }^{5}$ Department of Nephrology, Ankara Training and Research Hospital, Ankara, Turkey

\section{ABSTRACT}

Objectives: In our study, we investigated whether the undercarboxylated osteocalcin (ucOC) is an indicator of bone turnover for patients treated with hemodialysis (HD) or peritoneal dialysis (PD). Furthermore, we have examined the relationships between ucOC levels and other bone inidicators such as osteocalcin (OC), bone specific alkaline phosphatase (B-ALP), calcitonin, vitamin D, intact parathyroid hormone (iPTH), calcium $(\mathrm{Ca})$, phosphate $(\mathrm{P})$, magnesium $(\mathrm{Mg})$ and bone mineral density $(\mathrm{BMD})$.

Methods: Study group was consisted of $24 \mathrm{HD}, 30 \mathrm{PD}$ patients and 30 control subjects. ucOC measurements were based on precipitation of carboxylated OC with barium sulfate. After precipitation, ucOC was measured in supernatant by ELISA.

Results: In chronic kidney disease (CKD), increased ucOC levels were present both in HD and PD groups. The ucOC levels in HD group were higher than those of PD group. ucOC levels in samples after HD were lower than in samples before HD. But there is no difference between groups for ucOC\% levels. We observed that ucOC levels for CRF were higher compared to that of control group and statistically significant. ucOC levels were positively correlated with OC, B-ALP, ALP, iPTH, P and Mg levels. There were negative and significant correlations between ucOC levels and BMD values. ucOC has a good discrimination power for both high and low turnover ROD groups.

Conclusions: ucOC is a useful marker to evaluation of bone metabolism in patients undergone hemodialysis or peritoneal dialysis in end-stage renal disease.

Keywords: Renal bone disease, hemodialysis, peritoneal dialysis, undercarboxylated osteocalcin, osteocalcin, bone mineral density

$P$ atients with chronic kidney disease (CKD) have a wide spectrum of bone diseases, such as secondary hyperparathyroidism, osteomalacia, and adynamic bone disease [1]. Mineral and bone metabolism disorders in dialysis patients have been associated with car- diovascular calcifications, bone fractures, increased morbidity and mortality risks $[2,3]$. Therefore, it is important to identify bone and mineral disorders in patients with renal failure. Bone biopsies, scintigraphic screening studies, invasive and/or expensive proce- 
dures such as a computed tomography are used after double tetracycline labeling in the diagnosis of bone diseases [4]. For this reason, specific biochemical markers are needed which can be used to measure bone metabolism in both uremic and other diseases.

Serum osteocalcin (OC) is a bone formation marker, including three gamma carboxyglutamic (Gla) residues. Vitamin $\mathrm{K}$ is a cofactor for OC. OC is provided to be activated by vitamin $\mathrm{K}$-dependent carboxylation of these Gla residues. The non-gammacarboxylated form is called undercarboxylated osteocalcin (ucOC). Hydroxyapatite (HA) crystals cannot bind to ucOC and these results in changes in the bone matrix [5-7].

In the patients with $\mathrm{CKD}$, the relationship between ucOC and and chronic kidney disease-mineral and bone disorder (CKD-MBD) is not fully understood. ucOC may play a role in the pathogenesis of bone metabolism disorders that occur as a result of both vita$\min \mathrm{K}$ concentration changes and hyperparathyroidism in dialysis patients. In one study it was founded that suboptimal vitamin K deficiency in hemodialysis patients was associated with increased prevalence of hyperparathyroidism and increased risk of bone fracture. In this study, a significant inverse correlation was found between the parathyroid hormone (PTH) and vitamin K [8]. Although many bone markers have been studied in patients with $\mathrm{CKD}$, there are not enough studies in the literature showing the relation between ucOC and CKD-MBD.

ucOC concentration is measured indirectly by using the binding properties of barium sulfate (BS) or HA with osteoclastic glutamic acid residues. ucOCis stayed in the supernatant after carboxylated OC is precipitated by BS or HA. ucOC is measured with immunochemistry OC analysis methods [9]. ucOC concentration is also measured directly with ucOC specific ELISA methods [10].

The purpose of this study was to determine the relationship between serum UCOC concentrations and renal bone turnover in patients on hemodialysis (HD) and continuous ambulatory peritoneal dialysis (CAPD), and to determine the correlation of ucOC concentrations with bone mineral density (BMD), OC, alkaline phosphatase (ALP), bone-specific alkaline phosphatase (B-ALP), intact parathyroid hormone (iPTH), calcium $(\mathrm{Ca})$, ionized calcium (iCa), magnesium $(\mathrm{Mg})$, phosphate $(\mathrm{P})$ and vitamin D [25(OH)D3)] concentrations in patients and control samples. We also planned to compare indirectly measured ucOC concentrations using the binding properties of $\mathrm{OC}$, with BS.

\section{METHODS}

\section{Patient and Control Groups}

The study was conducted at the Biochemistry Department of Ankara Training and Research Hospital, Ministry of Health, with the support of the Nephrology Clinic. The study was approved by the local Ankara Training and Research Hospital Ethics Committee (Decision no: 2736/2009) and conducted in accordance with the principles of the Declaration of Helsinki. All patients were informed of the study and signed written approvals.

The patient group included a total of 30 patients for CAPD and 24 for HD. The control group included 30 healthy subjects free from hypertension, diabetes mellitus, thyroid disease, liver and renal diseases, and any other known disease. Dialysis patients that had any bone diseases or broken bones and diabetes mellitus were excluded from the study. Demographic properties of the patients are shown in Table 1.

In patients with $\mathrm{CKD}$, iPTH measurement is important in the diagnosis and follow-up of ROD. Simply, ROD was classificated by iPTH results. $<100$ $\mathrm{pg} / \mathrm{mL}$ and $>300 \mathrm{pg} / \mathrm{mL}$ iPTH concentrations were separated low-turnover bone disease and highturnover bone disease, respectively.

\section{Blood Samples}

Blood was collected from the HD patients before and after dialysis, and from the CAPD patients and control group in the morning before meals. Blood samples were drawn into evacuated serum separator tubes containing clot activator (SST Vacutainer ${ }^{\circledR}$, Becton Dickinson). All blood samples were centrifuged within 30 minutes, at $1500 \mathrm{~g}$ (rpm) for 10 minutes. Serum samples obtained were collected into Eppendorf tubes (Eppendorf $₫$ Safe-Lock microcentrifuge tubes, Turkey). The routine biochemistry analysis and $\mathrm{iPTH}$ were measured within the same day. 25(OH)D3, OC anducOC serum samples was stored in a deep freezer at $-80{ }^{\circ} \mathrm{C}$ until working day. 
Table 1. Descriptive characteristics of patient and control groups

\begin{tabular}{|c|c|c|c|}
\hline Variables & $\begin{array}{l}\text { CAPD patients } \\
\quad(\mathbf{n}=\mathbf{3 0}) \\
\end{array}$ & $\begin{array}{c}\text { HD Patients } \\
(\mathrm{n}=24)\end{array}$ & $\begin{array}{l}\text { Control } \\
(n=30) \\
\end{array}$ \\
\hline \multicolumn{4}{|l|}{ Gender (n, \%) } \\
\hline Female & $13(43.3 \%)$ & $8(33 \%)$ & $12(40 \%)$ \\
\hline Male & $17(56.7 \%)$ & $16(67 \%)$ & \\
\hline Age $($ years $)(x \pm S D)$ & $46.8 \pm 13.3$ & $49.2 \pm 15.3$ & $47.9 \pm 14.5$ \\
\hline BMI $\left(\mathbf{k g} / \mathbf{m}^{2}\right)(x \pm$ SD $)$ & $22.52 \pm 3.6$ & $22.58 \pm 3.2$ & $24.7 \pm 3.7$ \\
\hline KT/V & & $1.64 \pm 0.31$ & \\
\hline URR (Urea reduction rate), \% & & $71.47 \pm 7.29$ & \\
\hline \multicolumn{4}{|l|}{ Duration of initiating dialysis (year) } \\
\hline$<$ 1year & 5 & 7 & - \\
\hline 1-5years & 19 & 12 & - \\
\hline 5-10years & 6 & 5 & - \\
\hline \multicolumn{4}{|l|}{ Drugs used } \\
\hline Anti-lipidemic (\%) & $11 \%$ & $16 \%$ & - \\
\hline Antihypertensive (\%) & $65 \%$ & $38 \%$ & - \\
\hline T3 agonist & $9 \%$ & - & - \\
\hline Folic acid derivative $(\%)$ & $29 \%$ & $79 \%$ & - \\
\hline Iron drugs & $30 \%$ & $76 \%$ & - \\
\hline Phosphate binding & $45 \%$ & $91 \%$ & - \\
\hline Recombinant erythropoietin analogs & $11 \%$ & $78 \%$ & - \\
\hline Anti-aggregate drugs & $32 \%$ & $1 \%$ & - \\
\hline $\mathrm{D}$ vitamin analogs & $95 \%$ & $100 \%$ & \\
\hline
\end{tabular}

$\mathrm{HD}=$ hemodialysis, $\mathrm{CAPD}=$ continuous ambulatory peritoneal dialysis, $\mathrm{BMI}=$ body mass index

\section{Laboratory tests}

Analysis of serum glucose, urea, creatine, uric acid, ALP, B-ALP, Ca, Mg, and P were made with the original reagents in the Olympus AU 2700 (Mishima Olympus Co. Ltd. JAPAN). For B-ALP analysis the method described by Mass et al. [11] was used. ALP derived liver was measured in the supernatant after serum B-ALP was inhibited with urea. The obtained ALP values were subtracted from total ALP and BALP was determined. Serum iPTH was measured with the original reagents using the chemiluminescence enzyme immunoassay method in Advia Centaur (Siemens Diagnostic Products Corporation, LosAngeles, CA, USA). 25(OH)D3 was measured by DIA source 25OH-VIT. D3-RIA-CT reagent (DIA source IMMUNOassays S.A., Nivelles, Belgium) with methodin Gamma counter C12 NE 1600 (Bio DPC,
USA). OCwas measured by ELISA method using the osteocalcin BioSource Host-EASIA Kit (BioSource Europe SA, Nivelles, Belgium) in the Triturus ${ }^{\circledR}$ EIA (GRIFOLS, Los Angeles, USA) analyzer.

Measurements of Serum Undercarboxylated Osteocalcin with Barium Sulphate

50 mg BS (Sigma Chemical Co., St. Louis, Mo.) were weighed on a precision scale. Each one was placed in separate Eppendorf microcentrifuge (Eppen$\operatorname{dorf}^{\circledR}$ Safe-Lock microcentrifuge tubes, Turkey) tubes.

Serum samples stored at $-80^{\circ} \mathrm{C}$ were dissolved by leaving the mat room temperature and mixing them using a vortex mixer. $50 \mathrm{mg}$ of BS were mixed with $500 \mu \mathrm{l}$ of serum sample. The mixtures were vortexed and then stirred at $4^{\circ} \mathrm{C}$ for 30 minutes in the mixer. The carboxyl OC was then precipitated with BS by 
centrifugation at $2000 \mathrm{~g}$ for $15 \mathrm{~min}$ at $4^{\circ} \mathrm{C}$. The supernatants containing ucOC were transferred to another tube and these samples were run on the same day. ucOC concentrations were measured by the ELISA method using the OC BioSource Host-EASIA Kit (BioSource Europe SA, Nivelles, Belgium) in the Triturus $^{\circledR}$ EIA (GRIFOLS, Los Angeles, USA) analyzer.

Two separate serum pools were constructed containing low and high ucOC concentrations for the inter-day and intra-study precision of the ucOC measurement methods. The total coefficient of variation $(\mathrm{CV})$ was calculated for intra-assay $(\mathrm{n}=21)$ and between-days $(\mathrm{n}=25)$.

For the recovery work, the two pools were prepared at low and high ucOC concentrations for BS. The two pools were found to have $1.87 \mathrm{ng} / \mathrm{mL}$ and $16.28 \mathrm{ng} / \mathrm{mLucOC}$ concentrations for the BS precipitation method.

$\%$ Recovery $(\mathrm{R})$ was calculated by taking the average of recovery values obtained by mixing in ratio 1High $(\mathrm{H}) / 1 \mathrm{H}, 1 \mathrm{Low}(\mathrm{L}) / 1 \mathrm{H}, 1 \mathrm{H} / 2 \mathrm{~L}, 2 \mathrm{H} / 3 \mathrm{~L}, 3 \mathrm{~L} / 2 \mathrm{H}$, $1 \mathrm{~L} / 3 \mathrm{H}$, and $1 \mathrm{~L} / 2 \mathrm{H}$.

\section{Bone Mineral Density Measurement}

BMD measurements for the patient and control groups were done within a week after blood samples were received. BMD was measured by Dual Energy X-Ray Absorbtiometry (DEXA-hologic ${ }^{\circledR}$ QDR$4500 \mathrm{~W}$, Waltham, MA, USA). BMD is expressed as grams per square centimeter. All BMD measurements were performed in the supine position and obtained for the lumbar spine at the L1 through L4 level and for left hip measurement at the femur neck, trochanteric region, Wards triangle in the patients. Results for lumbar spinal BMD were expressed as the average of L1 through L4 values. The World Health Organization (WHO) definition was applied for osteoporosis (T-score $\leq-2.5 \mathrm{SD}$ ), osteopenia (T-score between -1.0 and $-2.5 \mathrm{SD}$ ) and normal BMD (T-score $\geq-1.0 \mathrm{SD}$ ) according to the $\mathrm{T}$ score of the lumbar spines (1-4.) and/or left femur neck [12].

\section{Dialysis Procedure}

All patients underwent hemodialysis three times per week for a duration of four hours a day, using a synthetic low-flux hollow-fiber filter (polysulfone membrane) (surface area $1.25 \mathrm{~m} 2$ ), a mean blood flow speed of 200-300 $\mathrm{mL} / \mathrm{min}$, and a dialysate flow speed of approximately $500 \mathrm{~mL} / \mathrm{min}$ during the HD procedure. For peritoneal dialysis patients, CAPD involves four or five dwells per day of 2-2.5 liters per dwell.

\section{Statistical Analysis}

Statistical analysis was performed using SPSS for Windows v15.00 software (SPSS Inc., Chicago, IL, USA). Descriptive data were expressed in mean (X), standard deviation (SD), median (M), interquartile range (IQR), while the Distribution of the groups was evaluated using the Kolmogorov-Smirnov test. Biochemical variables were not normally distributed. The differences between the control, CAPD and HD groups compared by Kruskal Wallis test. Bonferroni posthoc test performed after Kruskal Wallis test. BMD variables were normally distributed. The comparison of BMD variables was conducted one-way ANOVA test. Comparison of analyties concentrations of serum collected before and after HD was made using the Wilcoxon sing test. Correlations between ucOC and all variables (biochemical and BMD) were assessed by non-parametric Spearman's rank correlation test. A $p$ - value of $<0.05$ was considered statistically significant. ROC analysis was performed to determine the renal osteodystrophy (ROD) discrimination of ucOC and other parameters. Cut off was made $300 \mathrm{pg} / \mathrm{mL}$ iPTH to identify as high turnover bone ROD for ROC analysis $(n=26)$.

\section{RESULTS}

Both ucOC concentrations in the CAPD group were significantly higher than in the control group ( $p$ $<0.001$ ). There was no significant difference between the ucOC $\%$ values in the CAPD and control groups ( $p$ $=0.568$, base on posthoc comparison) (Table 2).

The ucOC concentrations in the HD group were higher than in the control group and the difference between the two groups was statistically significant $(p<$ $0.001)$. There was no significant difference between the HD and control groups in ucOC $\%$ values ( $p=$ 0.639 , base on posthoc comparison). The values of ucOC and other parameters measured in the patient and control group are shown in Table 2. Box plot graphs of ucOC levels in HD, CAPD and control groups are shown in Fig. 1.

Pre-HD ucOC levels were higher than post-HD. 
Table 2. Comparison of measured analytes of serum in the study groups

\begin{tabular}{|c|c|c|c|c|c|}
\hline Variables & $\begin{array}{c}\text { CAPD } \\
\text { patients } \\
(n=30)\end{array}$ & $\begin{array}{l}\text { Pre-HD } \\
\text { patients } \\
(n=24)\end{array}$ & $\begin{array}{l}\text { Post-HD } \\
\text { patients } \\
(n=24)\end{array}$ & $\begin{array}{l}\text { Control } \\
(n=30)\end{array}$ & $p$ value* \\
\hline $\mathrm{ucOC}, \mathrm{ng} / \mathrm{mL}$ & $6.73(5.19)$ & $9.82(11.99)$ & $4.46(3.57)$ & $0.29(0.21)$ & $<\mathbf{0 . 0 0 1}^{\mathrm{a}, \mathbf{b}, \mathbf{c}, \mathbf{d}}$ \\
\hline ucOC \% & $15.36(6.98)$ & $16.79(8.75)$ & $20.7(18.31)$ & $18.6(10.21)$ & $0.265^{\mathrm{c}}$ \\
\hline $\mathrm{OC}, \mathrm{ng} / \mathrm{mL}$ & $46.64(25.68)$ & $53.94(40.96)$ & $35.56(33.88)$ & $2.3(1.87)$ & $<0.001^{\mathrm{a}, \mathrm{b}, \mathrm{c}}$ \\
\hline B-ALP, U/L & $70(47.0)$ & $82(70.25)$ & $89.5(116.5)$ & $46(16)$ & $0.001^{\mathrm{a}, \mathrm{b}, \mathrm{c}}$ \\
\hline ALP, U/L & $102(34)$ & $98(82.5)$ & $105.0(128.5)$ & $65(16)$ & $<0.001^{\text {a,b,c }}$ \\
\hline $25(\mathrm{OH}) \mathrm{D}, \mathrm{ng} / \mathrm{mL}$ & $18.0(11.8)$ & $28.25(18.50)$ & & $28.19(23.40)$ & $0.598^{\mathrm{a}}$ \\
\hline iPTH, pg/mL & $328.1(225.1)$ & $266.5(230.0)$ & & $50.50(8.10)$ & $<0.001^{\mathrm{a}, \mathrm{b}}$ \\
\hline $\mathrm{Ca}, \mathrm{mg} / \mathrm{dL}$ & $9.08(1.00)$ & $8.94(1.03)$ & $10.37(1.38)$ & $9.40(0.50)$ & $<0.001^{\mathrm{a}, \mathrm{b}}$ \\
\hline $\mathrm{iCa}, \mathrm{mmol} / \mathrm{L}$ & $0.96(0.28)$ & $0.93(0.29)$ & $1.11(0.10)$ & $1.03(0.11)$ & $0.160^{\mathrm{c}}$ \\
\hline $\mathrm{P}, \mathrm{mg} / \mathrm{dL}$ & $4.53(1.31)$ & $4.48(2.40)$ & $2.75(1.25)$ & $3.01(0.60)$ & $<0.001^{\mathrm{a}, \mathrm{b}, \mathrm{c}}$ \\
\hline \multicolumn{6}{|l|}{$\mathrm{Ca} \times \mathrm{P}(\mathrm{mg} 2 / \mathrm{dl} 2)$} \\
\hline $\mathrm{Mg}, \mathrm{mmol} / \mathrm{L}$ & $1.0(0.1)$ & $1.05(0.10)$ & $0.80(0.08)$ & $0.81(0.10)$ & $<0.001^{\mathrm{a}, \mathrm{b}, \mathrm{c}}$ \\
\hline
\end{tabular}

Values are presented as median (inter quartile range). ${ }^{*}$ The differences between the control, CAPD and HD groups compared by Kruskal Wallis test. ${ }^{\mathrm{a} C}$ Comparison between control and CAPD, ${ }^{\mathrm{b}}$ Comparison between control and HD, ${ }^{\mathrm{c}}$ Comparison between Pre-HD and Post- HD, ${ }^{\mathrm{d}}$ Comparison between Pre-HD and CAPD. The same letters (a, b) indicate significant differences between groups base on Bonferroni posthoc comparison. $p$ - value $<0.05$ is statistically significant. $\mathrm{ucOC}=$ undercarboxylated osteocalcin, $\mathrm{HD}=$ hemodialysis, $\mathrm{CAPD}=$ continuous ambulatory peritoneal dialysis, $\mathrm{OC}=$ osteocalcin, $\mathrm{ALP}=$ alkaline phosphatase, $\mathrm{B}-\mathrm{ALP}=$ bone specific alkaline phosphatase, $\mathrm{PTH}=$ intact parathyroid hormone, $\mathrm{Ca}=$ calcium, $P=$ phosphate, $\mathrm{Mg}=$ magnesium

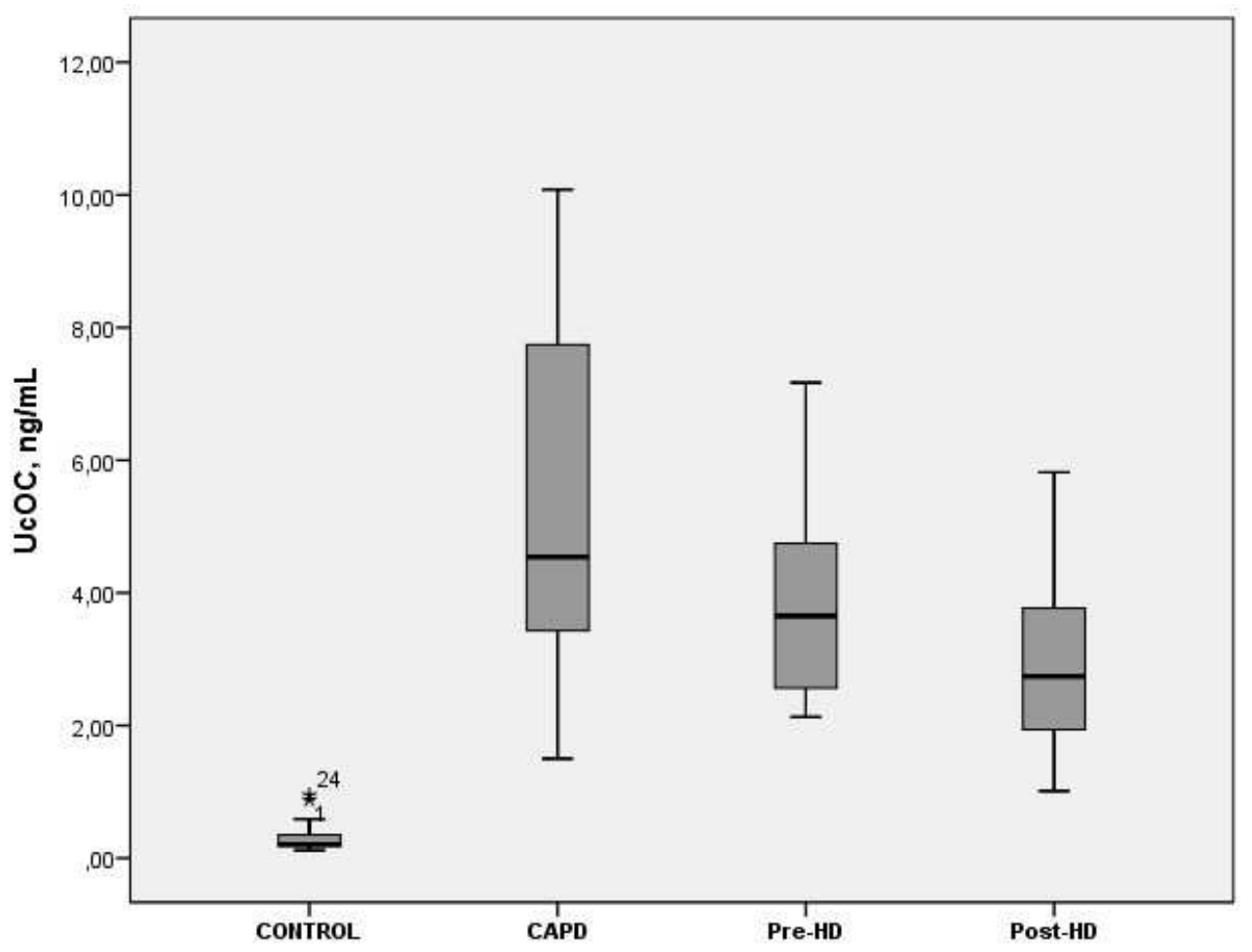

Fig. 1. ucOC levels in control, CAPD, pre-HD and post-HD groups. Box, inter quartile range (IQR): between 25th and 75th percentiles, horizontal line: median (M) 
Table 3. The correlation coefficients between ucOC and biochemical variables and BMD values in the study groups

\begin{tabular}{lcccc}
\hline & $\begin{array}{c}\text { CAPD patients } \\
(\mathbf{n}=\mathbf{3 0})\end{array}$ & $\begin{array}{c}\text { HD Patients } \\
(\mathbf{n}=\mathbf{2 4})\end{array}$ & $\begin{array}{c}\text { Control } \\
(\mathbf{n}=\mathbf{3 0})\end{array}$ & All groups \\
\hline Variables & $\mathbf{u c O C}$ & $\mathbf{u c O C}$ & $\mathbf{u c O C}$ & ucOC \\
\hline $\mathrm{ucOC}, \mathrm{ng} / \mathrm{mL}$ & $1.000^{*, \#}$ & $1.000^{*, \#}$ & 1.00 & $1.000^{*, \#}$ \\
$\mathrm{OC}, \mathrm{ng} / \mathrm{mL}$ & $0.781^{*}$ & $0.831^{*, \#}$ & 0.642 & $0.828^{*, \#}$ \\
$\mathrm{~B}-\mathrm{ALP}, \mathrm{U} / \mathrm{L}$ & 0.152 & 0.278 & 0.280 & $.456^{*}$ \\
$\mathrm{ALP}, \mathrm{U} / \mathrm{L}$ & 0.181 & 0.310 & 0.206 & 0.469 \\
\hline $25(\mathrm{OH}) \mathrm{D}, \mathrm{ng} / \mathrm{mL}$ & -0.181 & -0.027 & -0.206 & $-0579^{*}$ \\
$\mathrm{iPTH}, \mathrm{pg} / \mathrm{mL}$ & 0.059 & $0.600^{*}$ & 0.045 & $0.691^{*}$ \\
$\mathrm{Ca}, \mathrm{mg} / \mathrm{dL}$ & -0.145 & -0.200 & -0.083 & -0.148 \\
$\mathrm{iCa}, \mathrm{mmol} / \mathrm{L}$ & -0.186 & -0.29 & -0.174 & -0.043 \\
$\mathrm{P}, \mathrm{mg} / \mathrm{dL}$ & 0.232 & 0.005 & 0.069 & 0.348 \\
$\mathrm{Mg}, \mathrm{mmol} / \mathrm{L}$ & 0.215 & 0.115 & 0.203 & 0.492 \\
\hline $\mathrm{L} 1-\mathrm{L} 4, \mathrm{~g} / \mathrm{cm} 2$ & -0.168 & -0.241 & -0.176 & -0.115 \\
FemurTotal, g/cm2 & -0.149 & -0.104 & -0.367 & $-0.455^{*}$ \\
\hline
\end{tabular}

*Indicates statistically significant correlation $(p<0.05)$, \#Indicates statistically a strong significant correlation. BMD

$=$ bone mineral density, $\mathrm{ucOC}=$ undercarboxylated osteocalcin, $\mathrm{HD}=$ hemodialysis, $\mathrm{CAPD}=$ continuous ambulatory peritoneal dialysis, $\mathrm{OC}=$ osteocalcin, $\mathrm{ALP}=$ alkaline phosphatase, $\mathrm{B}-\mathrm{ALP}=$ bone specific alkaline phosphatase, $\mathrm{iPTH}=$ intact parathyroid hormone, $\mathrm{Ca}=$ calcium, $\mathrm{P}=$ phosphate, $\mathrm{Mg}=$ magnesium, $\mathrm{L}=$ Lumbar

This difference was statistically significant $(p<0.005)$. Although the \%UcOC levels were slightly higher in the pre-DH, there was no significant difference between the two groups.
There is significant strong correlation between ucOC and ALP, B-ALP and iPTH concentrations when all groups were combined. Table 3 are shown the correlations among ucOC and other biochemical and

Table 4. Comparison of BMD values in the study groups.

\begin{tabular}{|c|c|c|c|c|}
\hline $\begin{array}{l}\text { BMD } \\
(\mathrm{g} / \mathrm{cm} 2)\end{array}$ & $\begin{array}{l}\text { CAPD Patients } \\
\qquad(\mathrm{n}=\mathbf{3 0})\end{array}$ & $\begin{array}{l}\text { HD Patients } \\
\quad(n=24)\end{array}$ & $\begin{array}{l}\text { Control } \\
(\mathrm{n}=\mathbf{3 0})\end{array}$ & $P$ value* \\
\hline L1 & $0.870 \pm 0.16$ & $0.781 \pm 0.224$ & $1.02 \pm 0.205$ & $<0.001^{\mathrm{a}, \mathrm{b}}$ \\
\hline L2 & $0.896 \pm 0.237$ & $0.844 \pm 0.216$ & $1.087 \pm 0.204$ & $<0.001^{\mathrm{a}, \mathrm{b}}$ \\
\hline L3 & $0.948 \pm 0.218$ & $0.867 \pm 0.245$ & $1.121 \pm 0.202$ & $<0.001^{\mathrm{a}, \mathrm{b}}$ \\
\hline L4 & $0.965 \pm 0.192$ & $0.877 \pm 0.214$ & $1.125 \pm 0.196$ & $<0.001^{a, b}$ \\
\hline L1-L4 & $0.933 \pm 0.174$ & $0.846 \pm 0.212$ & $1.106 \pm 0.215$ & $<0.001^{\mathrm{a}, \mathrm{b}}$ \\
\hline Femur neck $\left(\mathrm{g} / \mathrm{cm}^{2}\right)$ & $0.734 \pm 0.118$ & $0.646 \pm 0.116$ & $0.919 \pm 0.152$ & $<0.001^{\mathrm{a}, \mathrm{b}, \mathrm{c}}$ \\
\hline Trochanteric region & $0.597 \pm 0.119$ & $0.518 \pm 0.137$ & $0.774 \pm 0.156$ & $<0.001^{\mathrm{a}, \mathrm{b}}$ \\
\hline Wards Triangle $\left(\mathrm{g} / \mathrm{cm}^{2}\right)$ & $0.651 \pm 0.133$ & $0.535 \pm 0.203$ & $0.738 \pm 0.146$ & $<0.001^{a, b}$ \\
\hline Femur Total & $0.835 \pm 0.136$ & $0.709 \pm 0.177$ & $0.989 \pm 0.159$ & $<0.001^{\text {a,b }}$ \\
\hline
\end{tabular}

Values are presented as mean \pm standart deviation. *one- way ANOVA test ${ }^{\mathrm{a} C o m p a r i s o n}$ between control and CAPD,

${ }^{b}$ Comparison between control and HD, ${ }^{c}$ Comparison between Pre-HD and CAPD The same letters $(a, b)$ indicate significant differences between groups base on Scheffe multiple comparison. $p$-value $<0.05$ is statistically significant. $\mathrm{BMD}=$ bone mineral density, $\mathrm{L}=$ Lumbar 
Table 5. AUC values of the variables in determining high-turnover ROD according to iPTH $(>300 \mathrm{pg} / \mathrm{mL})$ results

\begin{tabular}{|c|c|c|c|c|}
\hline \multirow[t]{2}{*}{ Variables } & \multirow[t]{2}{*}{$\mathbf{A U C}$} & \multirow[t]{2}{*}{ p value } & \multicolumn{2}{|c|}{ Asymptotic $95 \%$ Confidence Interval } \\
\hline & & & Lower bound & Upper bound \\
\hline $\mathrm{ucOC}$ & 0.869 & $<0.001$ & 0.785 & 0.953 \\
\hline $\mathrm{ucOC} \%$ & $<0.5$ & 0.060 & 0.299 & 0.575 \\
\hline $\mathrm{OC}$ & 0.875 & 0.001 & 0.795 & 0.956 \\
\hline B-ALP & 0.783 & 0.001 & 0.669 & 0.897 \\
\hline ALP & 0.815 & 0.001 & 0.702 & 0.927 \\
\hline $25(\mathrm{OH}) \mathrm{D}$ & $<0.5$ & 0.771 & 0.099 & 0.332 \\
\hline L1-L4 & $<0.5$ & 0.251 & 0.344 & 0.616 \\
\hline FemurTotal & $<0.5$ & 0.286 & 0.212 & 0.474 \\
\hline
\end{tabular}

$\mathrm{AUC}=$ Area under curve, $\mathrm{ucOC}=$ undercarboxylated osteocalcin, $\mathrm{OC}=$ osteocalcin, $\mathrm{ALP}=$ alkaline phosphatase, $\mathrm{B}-$ $\mathrm{ALP}=$ bone specific alkaline phosphatase, $\mathrm{ROD}=$ renal osteodystrophy, $\mathrm{iPTH}=$ intact parathyroid hormone

BMD variables.

When compared to the control groups, BMD values were found to be statistically and significantlyhigher than in both the HD andCAPD groups. The BMD values are shown in Table 4.

Patients were classified according to iPTH concentrations $(n=84)$ to identify high turnover ROD. Patients with iPTH $>300 \mathrm{pg} / \mathrm{mL}$ were identified as high turnover bone ROD $(\mathrm{n}=26)$. Cut off was made $300 \mathrm{pg} / \mathrm{mL}$ iPTH for ROC analysis. The area under curve (AUC) values of the ROC analysis of the variables is shown in Table 5.

Precision results in our study were as follows: variation coefficients $(\mathrm{CV})$ of intra- assays for ucOC were $9.1 \%$ for $1.86 \mathrm{ng} / \mathrm{mL}$ and $5.11 \%$ for 16.13 $\mathrm{ng} / \mathrm{mL}$; between-days for CVwere $10.32 \%$ for 1.86 $\mathrm{ng} / \mathrm{mL}$ and $5.16 \%$ for $16.13 \mathrm{ng} / \mathrm{mL}$. The average $\% \mathrm{R}$ was $94 \%$ for BS. The $\%$ recovery values are shown in Table 6.

\section{DISCUSSION}

In our study, we aimed to determine the relationship between serum ucOC concentrations and renal bone turnovers in hemodialysis and peritoneal dialysis patients. It is also comparable to indirectly measured ucOC concentrations by taking advantage of the BS and HA binding properties of OC. In our study, it was determined that $\mathrm{uCOC}$ concentrations were high in pa-

Table 6. Recovery (\%) values of ucOC

\begin{tabular}{lccc}
\hline & Expected value $(\mathbf{n g} / \mathbf{m L})$ & Measured value $(\mathbf{n g} / \mathbf{m L})$ & R $(\%)$ \\
\hline & ucOC & ucOC & ucOC \\
\hline $\mathrm{H} / 1 \mathrm{H}$ & 16.28 & 15.07 & 92 \\
\hline $\mathrm{H} / 1 \mathrm{~L}$ & 11.75 & 12.01 & 114 \\
$3 \mathrm{H} 2 \mathrm{~L}$ & 10.49 & 9.08 & 86 \\
$1 \mathrm{H} / 1 \mathrm{~L}$ & 9.07 & 8.19 & 90 \\
$2 \mathrm{H} / 3 \mathrm{~L}$ & 7.03 & 6.19 & 84 \\
$1 \mathrm{H} / 2 \mathrm{~L}$ & 6.60 & 7.19 & 108 \\
$1 \mathrm{~L} / 1 \mathrm{~L}$ & 1.87 & 1.67 & 89 \\
Total R\% & & & 94 \\
\hline
\end{tabular}

$\mathrm{L}=$ Low, $\mathrm{H}=$ High, ucOC $=$ undercarboxylated osteocalcin 
tients with CKD than those in the healthy control groups. Also, the ucOC concentrations in the HD group were higher than in the CAPD group. ucOC showed a good correlation with other bone formation markers ALP, B-ALP and iPTH. When iPTH with a value $>300 \mathrm{pg} / \mathrm{mL}$ was taken as a cut-off, ucOC concentrations for determining high-turnover ROD had a better value than other measured bone markers.

The relationship between ucOC and patients with CKD is not fully understood. In a study carried out by Kohmeier et al. [13], the role of ucOC in bone metabolism, the relationship between ucOCand vitamin K, the old fracture bone story, and the risk of bone fracture was investigated in patients with renal failure. In the study, the relation between bone fracture risk and high hyperparathyroidism prevalence with increased ucOC concentrations due to vitamin $\mathrm{K}$ deficiency was shown in HD patients. In a study carried out by Nagata et al. [14], serum ucOC was found to be significantly lower in pre-dialysis patients with chronic kidney disease (CKD) than in HD. In their study, there are significant correlations between ucOC and eGFR levels in pre-dialysis CKD patients [14]. In a the study carried out by Kuzniewski et al. [15], the serum ucOC concentrations in HD patients were significantly higher than in healthy subjects. These studies showed significant correlation between ucOC and iPTH. Our study was consistent with the results of these studies. In our study, the serum ucOC concentration was higher in the HD group than in the healthy control group. There was also a significant correlation between ucOC and OC, B-ALP and ALP concentrations in the HD group. When all study groups were combined, there was significant correlation between ucOC and $\mathrm{PTH}$ concentrations. Increasing $\mathrm{PPTH}$ concentrations in patients with CKD may have a positive effect on ucOC concentrations. Or conversely, ucOC elevation may be contributing to PTH elevation.

When we investigated ucOC concentrations in a CAPD patients group, the subclinical vitamin K deficiency and resulting changes in ucOC concentrations due to $\mathrm{K}$ vitamin status was studied [16]. In their study, the cut off for ucOC\% ratio was described to be $>20 \%$ in ROC analysis for determine high turnover ROD according for iPTH ( $>300 \mathrm{pg} / \mathrm{mL}$ ) concentrations. This study showed positive correlation between phosphate, iPTH and ucOC\%. In another in a study, theucOC/OC ratio was significantly higher in HD than in pre-dialysis patients with CKD [14]. In our study there wasn't a significantly different ucOC\% when all the groups were compared.

There are many factors thatcan affect bone metabolism in patients with CKD. High PTH concentrations, hypocalcemia, hyperphosphatemia, low vitamin $\mathrm{D}$ and many uremic toxins affect bone formation and destruction. There is therefore a marked increase in the markers of bone formation and destruction. In our study, ucOC concentration was high in patient groups. However, the lack of statistical difference between ucOC\% values suggests that not only UCOC but also OC concentrations are increased in HD and CAPD patients.

In our study, we measured ucOC before and after HD to investigate the effect of dialysis process on ucOC. Pre-HD ucOC levels were higher than postHD. This difference was statistically significant $(p<$ $0.005)$. Although the \%ucOC levels were slightly higher in the pre-DH, there was no significant difference between the two groups. According to this result, there is a decrease in ucOC concentrations due to the dialysis procedure. There is no information on the effect of dialysis in the literature search. Our prediction may be loss of ucOC or an increase in OC fragment due to dialysis.

OC is normally a molecule that undergoes rapid glomerular filtration. Increase in OC concentration is expected in renal failure. In uremic patients, osteoblastic activity is increased due to hyperparathyroidism, and consequently, $\mathrm{OC}$ is increased. It is indicated that different fragments of osteocalcin are released from bone tissue in renal failure [17]. Furthermore, different OC fragments occur due to proteolytic degradation of the OC molecule in the plasma. In CRF patients, $26 \%$ of total OC has been shown to be intact OC. The remaining $74 \%$ are reported to be composed of N-terminal, mid-region, mid-region $\mathrm{C}$-terminal and $\mathrm{C}$-terminal regions, which are mainly OC fragments. In the measurement of osteocalcin, these fragments may also contribute to high outcome [18]. In addition, the epitopes used in antibody production for OC measurements are important. In our study, the $\mathrm{OC}$ reagent used for detecting intact OC. This reagent used develops monoclonal antibodies against different epitopes of the molecule. The regions of these epitopes were not specified in the prospectus of the $\mathrm{OC}$ reagent.Thus, the ELISA reagent used for OC may have been measuring fragments that were outside the "intact" mole- 
cule in patients with CKD. This explains why ucOC\% does not differ between groups. If the different fragments of OC are measured, the increase in ucOC in the patient groups will not reflect the increase in ucOC\%.

In patients with CKD, secondary hyperparathyroidism is associated with the development of ROD and $\mathrm{PTH}$ measurement is important in the diagnosis and follow-up of ROD $<100 \mathrm{pg} / \mathrm{mL}$ and $>300 \mathrm{pg} / \mathrm{mL}$ iPTH concentrations suggest low-turnover bone diseaseand high-turnover bone disease, respectively [18]. In our study, iPTH concentrations were found to be $>$ $300 \mathrm{pg} / \mathrm{mL}$ in 22 patients, $100-300 \mathrm{pg} / \mathrm{mL}$ in $22 \mathrm{pa}-$ tients and $<100 \mathrm{pg} / \mathrm{mL}$ in 5 patients. When iPTH with a value $>300 \mathrm{pg} / \mathrm{mL}$ was taken as a cut-off, ucOC concentrations when determining high-turnover ROD according to PTH results had a better value than other measured bone markers. However, ROD is the gold standard of bone biopsy in diagnosis and typing. Therefore, evaluation based on bone biopsy is necessary. There is insufficient information in literature to determine the relationship between ROD and ucOC, which is the basis of bone biopsy. Therefore, further work is needed to determine the relationship between ROD and ucOC.

\section{Relationship between BMD values and bone mark- ers in CKD}

DEXA is a cheap, rapid and non-invasive technique for determining osteopenia. Although the value of BMD is low in determining the ROD type, it is used to show the bone mineral status of patients with CKD. It is known that kidney disease is a well-established risk factor for many changes in bone mineral metabolism and development of osteoporosis [19]. In our study, the prevalence of osteopenia and osteoporosis in the CKD subjects was $73 \%$ and $20 \%$, respectively. In a study by Rix et al. [20], BMD and biochemical bone markers were measured in patients with CKD. In this study, the BMD values of the patient group were found to be significantly lower than in the control group. In our study, the BMD values of CKD patients were statistically significantly lower compared to the control group. There is no study showing the relationship between BMD and ucOC concentration in patients with CKD. In other studies [22-24], there was a negative correlation between ucOC concentration and BMD. Similar to these studies in our study, a strong negative correlation was found between ucOC concentrations and BMD values in all patients and control groups.

\section{CONCLUSION}

ucOC concentrations in the CKD group were higher than those in the healthy control groups. Also, the ucOC concentrations in the HD group were higher than in the CAPD group. HD affects ucOC concentrations. ucOC\% levels, unexpectedly, did not differ between groups. This may be due to the increased number of OC fragments in the serum of the CKD patients. ucOC showed a good correlation with other bone formation markers ALP, B-ALP and iPTH. This suggests that ucOC may be a good bone marker in CRF. ucOC also showed a good discrimination in high and low alternating ROD groups compared to $\mathrm{iPTH}$ cut-off concentrations.

\section{Authors' Contribution}

Study Conception: MA, DY; Study Design: MA, DY; Supervision: MA, VF, DY; Funding: MA, GS, DY; Materials: MA, VF, AA, MD, DY; Data Collection and/or Processing: MA, VF, AA, MD; Statistical Analysis and/or Data Interpretation: MA, MFA, DY; Literature Review: MA, GS, MD, DY; Manuscript Preparation: MA, MFA and Critical Review: MA, DY.

\section{Conflict of interest}

The authors disclosed no conflict of interest during the preparation or publication of this manuscript.

\section{Financing}

The authors disclosed that they did not receive any grant during conduction or writing of this study.

\section{REFERENCES}

1. Kidney Disease: Improving Global Outcomes (KDIGO) CKDMBD Update Work Group. KDIGO 2017 Clinical Practice Guideline Update for the Diagnosis, Evaluation, Prevention, and Treatment of Chronic Kidney Disease-Mineral and Bone Disorder (CKD-MBD). Kidney Int Suppl (2011) 2017;7:1-59.

2. Dhondup T, Qian Q. Electrolyte and acid-base disorders in chronic kidney disease and end-stage kidney failure. Blood Purif 2017;43:179-88. 
3. Piraino B, Chen T, Cooperstein L, Serge G, Puschett J. Fractures and vertebral bone mineral density in patients with renal osteodystrophy. Clin Nephrol 1998;30:57-62.

4. Hutchison AJ, Whitehouse RW, Boulton HF, Adams EJ, Mawer BE, et al. Correlation of bone histology with parathyroid hormone, vitamin D3 and radiology in end-state renal disease. Kidney Int 1993;44:1071-7.

5. Vermeer C, Jie KSG, Knapen MHJ. Role of vitamin K in bone metabolism. Annu Rev Nutr 1995;15:1-22.

6. Saupe J, Shearer MJ, Kohlmeier M. Phylloquinone transport and its influence on gama -carboxyglutamate residues of osteocalcin in patients on maintenance hemodialysis. Am J Clin Nutr 1993;58:204-8.

7. Sokoll LJ, Sadowski JA. Comparison of biochemical indexes for assessing vitamin $\mathrm{K}$ nutritional status in a healthy adult population. Am J Clin Nutr 1996;63:566-73.

8. Malyszko J, Wolczynski S, Skrzydlewska E, Malyszko JS, MysliwiecM.Vitamin K status in relation to bone metabolism in patients with renal failure. Am J Nephrol 2002;22:504-8.

9. Sokoll L, O’Brien ME, Camilo ME, Sadowski JA. Undercarboxylated osteocalcin: development of a method to determine vitamin K status. Clin Chem 1995;41:1121-8.

10. Koyama N, Ohara K, Yokota H. A one step sandwich enzyme immunoassay for gamma-carboxylated osteocalcin using monoclonal antibodies. J Immunol Methods 1991;139:17-23.

11. Mass DW, Henderson AR, Enzymes: Burtis CA, Ashwood ER (Ed.) Tietz Textbook of Clinical Chemistry and Molecular Diagnostics. 2th ed. St. Louis, MO: Elsevier Saunders, 1994:843. 12. Fischer M, Raue F. Measurements of bone mineral density. Q J Nucl Med 1999;43:233-40.

13. Kohlmeier M, Saupe J, Shearer MJ, Schaefer K, Asmus G. Bone health of adullt hemodialysis patients is related $\mathrm{K}$ status. Kidney Int 1997;51:1218-21.

14. Nagata Y, Inaba M, Imanishi Y, Okazaki H, Yamada S, et al. Increased undercarboxylated osteocalcin/intact osteocalcin ratio in patients undergoing hemodialysis. Osteoporos Int
2015;26:1053-61.

15. Kuzniewski M, Fedak D, Dumnicka P, Kapusta M, Stępien E, Chowaniec, et al. Carboxylated and intact osteocalcin predict adiponectin concentration in hemodialyzed patients. Renal Fail 2016;38:451-7.

16. Gundberg CM, Weinstein RS. Multiple immunnoreactive forms of ostecalcin in uremic serum. J Clin İnvest 1986;77:176267.

17. Garneo P, Grimaux M, Seguin P, Delmas PD. Characterization of immunreactive forms of human osteocalcin generated in vivo and in vitro. J Bone Miner Res 1994;9:255-65.

18. Liach F, Bover J. Renal osteodystrophy in the kidney. In:BrennerBM, Rector FC, Eds. Brenner and Rector's kidney. Philedelphia, WB Saunders, 1996. p. 2187- 273.

19. Stehman-Breen CO, Sherrard DJ, Alem AM, Gillen DL, Heckbert SR, Wong CS, et al. Risk factors for hip fracture among patients with end-stage renal disease. Kidney Int 2000;58:22005 .

20. Rix M, Andreassen H, Eskildsen P, Langdahl B, Olgaard K. Bone mineral density and biochemical markers of bne turnover in patients with predialysis chronic renal failure. Kidney Int 1999;56:1084-93.

21. Szulc P, Chapuy MC, Meunier PJ, Delmas PD. Serum undercarbaxylated osteocalcin is a marker of risk of hip fracture in elderly women. J Clin Invest 1993;91:1769-74.

22. Kim SM, Kim KM, Kim BT, Joo NS, Kim KN, Lee DJ, et al. Correlation of undercarboxylated osteocalcin (ucOC) concentration and bone density with age in healthy Korean women.J Korean Med Sci 2010;25:1171-5.

23. Yamauchi M, Yamaguchi T, Nawata K, Takaoka S, Sugimoto T. Relationships between undercarboxylated osteocalcin and vitamin $\mathrm{K}$ intakes, bone turnover, and bone mineral density in healthy women. Clin Nutr 2010;22:434-56.

24. Plantalech L, Guillaumont M, Leclercq M, Delmas PD. Impaired carboxylation of serum osteocalcin in elderly women. J Bone Miner Res 1991;6:1211-16. 